\title{
Assessment of the peak-to-average power ratio in different channel organization strategies
}

\author{
Eduard Bertran \\ Department of Signal Theory and Communications, Universitat Politècnica de Catalunya (UPC) \\ c/ Esteve Terrades 7, Castelldefels, Barcelona, 08860, Spain.
}

e-mail: bertran@tsc.upc.edu

\begin{abstract}
The benefits of organizing multichannel communications by fragmenting the data in bonded or in aggregated channels have been usually assessed at the MAC (Media Access Control) layer, being the network traffic the purpose of the studies. Despite the importance of the different kinds of channel organizations over the transmit signal PAPR (Peak-to Average Power Ratio) to size the back-off value of the power amplifier, little attention has been paid to it. In this paper the key aspect is the PAPR, which is fundamental to face transmitter designs optimizing the trade-off between linearity and the time-oflife of the batteries. The results are of interest not only in ad-hoc cognitive radio networks, but also in some of the latest ETSI (European Telecommunications Standards Institute) and IEEE (Institute of Electrical and Electronics Engineers) standards supporting mobility in either WLAN (Wireless Local Area Network) environments or in cellular communications. A standardized radio link is used to model a realistic scenario to study different settings of the aggregate channels and to compare them with channel bonding alternatives. Besides, the suitability of some crest factor reduction (CFR) techniques is here considered for different channel organizations. The results show how the channel organization (contiguous, regular and random spaced), either with bonded or aggregated strategies, affects the PAPR.
\end{abstract}

Keywords PAPR. Power amplifiers. Channel bonding. Aggregation. CFR.

\section{Introduction}

In a number of modern communication standards, as well as in other specific developments not completely regulated yet, the transmit data is split over a number of channels (or carriers) allocated in different frequency bands, which may or may not be contiguous elements of the spectrum. In a multichannel system, the channel's operation is identified as bonded when it is adjacent to each other. Hence, channel bonding is a technique for contiguous channels, where all of them are merged into a single wideband one. Otherwise, if the occupied channels are not contiguous and jointed the operation mode is called aggregated, thus depending the terms bonding and aggregation on the way that the channels are allocated in the frequency spectrum. Both cases follow the same objective: to have a wider bandwidth, which should produce an increase of the data rates. Both techniques pursuit to reduce the channel traffic, mitigating the congestion. This way some MAC protocols have been proposed aiming at lower packet collisions and, consequently, higher data throughput [1].

In the LTE Advanced standard it is considered a spectrum capable of supporting scalable bandwidths by using spectrum aggregation when possible. While in LTE Release 8 the channels have a maximum bandwidth of 20 $\mathrm{MHz}$, in LTE Release 10 (LTE-Advanced) the transmission bandwidth can be extended by means of signaling mechanisms enabling aggregation of multiple carriers, and it can support up to five $20 \mathrm{MHz}$ aggregated component carriers, offering a peak data rate of more than $1 \mathrm{~Gb} / \mathrm{s}[2$, 3]. Within the carrier aggregation techniques, two main methodologies are permitted: intra-band and inter-band aggregation. In intra-band a single broadband is used, producing a wider contiguous channel by aggregating carriers adjacent to each other. This scenario is similar to the channel bonding situation. A mixed situation within the intra-band aggregation, which adds some additional complexity, is produced when a non-contiguous sub-carrier aggregation technique is used inside a unique broadband channel. On the other hand, the inter-band aggregation methodology takes advantage of the band fragmentation. This technique may require the use of multiple transceivers if the bands are too separated, or a single broadband amplifier if the bands are not excessively distant and the amplifier gain does not have to be very high (because of practical stability concerns). Apart from the obvious impact on cost, performance and consumed energy, as well as synchronization issues, the different multicarrier structures have to face additional requirements regarding 
intermodulation and cross modulation among the transceivers. Behind the basic operation of LTE networks, some works [4] have proposed ways to provide improvements of the throughput, to reduce the number of user outages, and to enhance spatial reuse.

There have been also proposed quasi-bonding schemes [5], where the channels are partially overlapped. This scheme is used in some wireless mesh networks scenarios, looking for a near-optimal performance.

Also, some elements of the IEEE 802.11 family of standards consider the use of channel bonding or aggregation techniques. The standard 802.11 ac operates in the $5 \mathrm{GHz}$ band, and the bandwidth may be of $80 \mathrm{MHz}$ by bonding two adjacent $40 \mathrm{MHz}$ channels. Moreover, by using two $80 \mathrm{MHz}$ channels, adjacent or not, the bandwidth may be optionally increased up to $160 \mathrm{MHz}$. In the $802.11 \mathrm{~s}$ standard it is an amendment for mesh networking where several non-overlapping channels are used for multichannel transmission. It uses a so-called coordination window for negotiating the use of the channels for data exchange, which are not required to be neighboring $[6,7]$.

Cognitive radio (CR) techniques based on the channel bonding technique (with spectrum management often based on a dynamic spectrum access) are used both in ad-hoc and standardized systems. CR networks may use single or multichannel sharing: in [8] the whole frequency range is split into multiple subchannels, being each subchannel shared by multiple users. What pursuits the standard IEEE 802.22 is the use of white spaces (unused spectrum) in the conventional analogue TV frequency spectrum. It may operate at frequencies ranging from $54 \mathrm{MHz}$ to $862 \mathrm{MHz}$, and it is able to utilize more than one channel at a time to provide the required throughput. A base station maintains and updates a list of backup channels, seamlessly switching among them to maintain the QoS for the 802.22 users. Other studies are also addressed to design minim access control protocols for digital TV broadcast (DTVB) white spaces [9]. Also, in 802.22, the use of cognitive communications has been proven as a tool to secure the spectrum sensing process against attackers [10].

The internet of things (IoT) takes advantage of some IEEE standards, such as the 802.15.4 (with up to 16 channels in the $2.4 \mathrm{GHz}$ band). It has been shown that a way to increase the MAC layer capacity is to exploit the multiple communication channels provided by the standard, such as the time slotted channel hopping MAC [11].

Applications like wireless sensor networks (WSN), or machine-to-machine communications (M2M), link data collectors of the same type into sub-networks, and hybrid channel assignment schemes have been proposed [12] WSN schemes have energy scarcity, so the sensor nodes have to be low-powered. WSN are just an example of the green radio networks [13] where communication schemes to improve the energy efficiency of the network, take advantage of the sporadic transmissions combined with a data aggregation process.

Data aggregation has not to be confused with the channel aggregation technique assessed below in this paper for different channel organizations [14]. Both kinds of aggregations (data and channel) may operate together, as is the case of the Delay Tolerant Networks [15,16], where data is aggregated (i.e, a social graph is constructed by aggregating all historical contact information), while the channels may be aggregated at the transport layer by multiplexing a data stream across multiple channels.

Apart from the above-mentioned ETSI or IEEE standards, some radio links such as the ones described in EN 302217 [17] describe aspects of the equipment and the antennas for service applications at different microwave bands. In coherence with CEPT and ITU-R recommendations some channel aggregations are permitted, subject to the channels availability and national license restrictions. In the higher bands of these standards, $56 \mathrm{MHz}$ channels are built by aggregating $28 \mathrm{MHz}$ ones, a fact also considered in CEPT/ERC/ Recommendation 12-02 E. In specific scenarios up to 4 channels are assembled in a single composite bitstream [17]. This last standardized radio-link will be specially considered in this paper in order to carry out the study on a true scenario.

The main purpose of this paper is to contribute to the assessment of the PAPR of different channel organization modes, given its fundamental role in transmitter designs aiming to optimize the trade-off between linearity and the time-of-life of the batteries. Next Section 2 gives a brief overview of some studies made at the MAC layer to assess the pros and cons of different channel organizations regarding data throughput, delays, traffic congestion, safety or disturbances to other channels. In Section 3 the relationship between the form of the multichannel organization and the consumption of the power amplifier is introduced. Section 4 presents the results of the simulation studies, based on the aforementioned standardized scenario, and considering different channel organizations with different numbers of active channels and relative placements among them. What is assessed in Section 5 is the suitability of some basic peak factor reduction techniques for both bonded and aggregated situations. And finally, Section 6 summarizes and concludes.

\section{Studies on multichannel operation}

Apart from studies conducted to discover spectrum holes and to investigate their utilization aiming at decreasing the contention on channels, minimizing interferences and improving the average channel efficiency [18], most of the published works studying the multichannel operation assess the performance of both channel bonding and aggregation techniques just at the MAC level [19].

For ad-hoc CR, some conditions under which it is beneficial to bond multiple channels for OSA (Opportunistic Spectrum Access) networks are assessed in [20], where it is proven that the benefits are not 
unconditional, depending on the Primary Users (PU) activity and/or the size of the PU channel pools. It is concluded that the benefits are more prominent when the PU activity is low. Dynamic channel aggregation and spectrum adaptation are useful features that increase the capacity of the secondary network (Secondary Users, SU) in heterogeneous traffic environments, but they can produce a throughput loss in PU if their activity is high. Similar conclusions have been presented in [21]. In [22] both the blocking probability and the average queuing delay have been considered in order to evaluate different dynamic channel access techniques in CR networks. What is proposed in [23] is a channel allocation scheme which can significantly improve the multimedia transmission performance of priority-based SUs.

Spectrum overlay exploits unused (white) spectral regions while spectrum underlay exploits underused (gray) spectral regions. According to [24] underlay uses more spectrum than overlay approaches and operates below the noise floor of PUs. Besides, underlay-CR waveform is able to adapt its bandwidth regarding user requirements and environmental conditions.

Bonding and aggregation have been also compared in [7], by considering packet collisions. In this work it is shown that in CSMA scenarios, the multichannel technique can improve both throughput and delay in high traffic conditions. The given reason is that, when channel traffic is high, there may be lots of packet collisions in the channelbonding network. So, if multiple channels are used to disperse the network traffic, the probability of packet collision can be reduced. In consequence, the multichannel technique can improve the delay performance when channel traffic is high, even though this result is also sensitive to the chosen access protocol.

A significant effort has been made to find optimum channel assignment algorithms, mainly for cellular communications, by using algorithms based on heuristic and/or genetic strategies. Recently, in [25] it has been formulated the joint power control and channel assignment optimization problem. In [26] two channel-assembling strategies which consider simultaneously spectrum adaptation and heterogeneous traffic are proposed. Channel assembling is proved as beneficial for elastic traffic (not requiring real-time operation) because of its flexibility, and a poorer overall performance is shown in the static strategy (spectrum handover only), except for a higher average service rate per ongoing SU service. Hence, a dynamic strategy (spectrum handover together with an adjustment of the number of the assembled channels for ongoing SU service) is recommended as the most appropriate alternative for channel assembling in multichannel CR networks.

Other works address the multichannel security. What is analyzed in [27] is the effect of a malicious attacker trying to create service disruption. In coherence with [21], it has been estimated that the attacker can cause up to $16 \%$ loss in the capacity of the system as a consequence of channel fragmentation. The capacity loss is increased as the degree of fragmentation grows. Besides, correlations between multiple pairs of fragments, which become stronger as the amount of fragmentations increase, enable an attacker to easily disturb more fragments and hence to increase the probability of service disruption.

Apart from the previous ad-hoc networks, the comparison of channel bonding and aggregation methods, at the MAC layer, has also been made over standardized frameworks. Examples are [27] and [28] where the study is carried out over the IEEE 802.22 standard. It has been shown that in case of channel aggregation done at the CPEs (Customers Premise Equipment) level, the transmission through one channel may cause RF problems to the adjacent ones, and even prevent their correct reception when the aggregated channel is transmitting, and occasionally violating the mandatory spectral protection. This issue is not so easily encountered when channel bonding is used. These last results reveal the higher importance of the selectivity (transmit power mask) of the power amplifiers, especially in the channel aggregation case.

In summary, from the existing simulation studies, it is not possible to conclude a definitive diagnosis on the advantages of channel bonding over channel aggregation, or vice versa. The preferred scenarios for channel bonding are for low PU channel activity in CR, as well as for elastic traffic. However, these conclusions may be modified if the number of packet collisions is high. At the physical layer, the channel bonding strategy allows better control of the resource allocation and the transmit power. Conversely, the transmit power mask is more critical for channel aggregation. Although, channel aggregation has been presented as a more delicate option in front of an attacker trying to disrupt the communications, this fact is also too sensitive to other factors, such as the way the information is split over the aggregated channels. Preferred scenarios for channel aggregation are for high SU activity (in CR) and for reducing packet collision in high traffic conditions.

\section{Effects of the multichannel strategy on the power amplifier biasing}

Among the physical subsystems constituting the transmitter, power amplifiers (PA) are the most critical ones to allow multichannel operation. The reason is twofold: on the one hand the PA is the most power hungry device among the transmitter subsystems, with a significance of $70-80 \%$ in mobile equipment. On the other hand, the stringent ACPR (Adjacent Channel Power Ratio) figures, necessary for multichannel operation, depend on the PA transmit mask, which cannot be improved by placing a posterior conventional filter (before the antenna) because of the required $\mathrm{Q}$ factor of this hypothetical filter.

The selectivity of the transmit power mask is especially important in channel aggregation to avoid spectral regrowth capable to block the receivers in the adjacent channels and, from an industrial perspective, to obtain the mandatory transmitter certification. If a bonding strategy requiring a composite broadband channel is chosen, it easily appears the typical compromise between bandwidth and stability in the PAs. Besides, the PAs have to meet the EVM (Error Vector Magnitude) requirement to also keep the standard's specifications, thus being a compromise between signal 
quality and power consumption. As a sample of such demanding requirements for the sake of linearity, we simply have to see the required EVM for 256-QAM modulations in some IEEE 802.11 standards, which is $2.5 \%$ $(-32 \mathrm{~dB}),[29]$

The aforementioned ACPR and EVM parameters depend on the signal PAPR, which is also the main point to face the trade-off between the linearity and the powerconsumption. PAPR depends on different aspects of the communication system such as the modulation format, the roll-off of the ISI filters and, moreover, the channel access method. High PAPR values require higher baked-off amplifiers, a solution that can lead to the use of the energetically inefficient class $\mathrm{A}, \mathrm{AB}$ or $\mathrm{B}$ PAs. An excessive focus in signal quality (i.e., increased back-off operation in the PA in order do not clip the signal peaks) may compromise battery life. Conversely, to enlarge the batteries time-of-life may degrade the signal performance, which has consequences in data rate (in-band distortion) and spectral regrowth.

As the data rate is increased, the quality of the signal becomes even more important. Current solutions to face high PAPR signals, avoiding the use of inefficient power amplifier schemes, may roughly be classified in three ways: the use of linearizers (i.e., digital predistorters, DPD), the previous signal conditioning (i.e, peak reduction, PR, techniques) or the use or efficient amplifiers structures, such as the polar transmitter or the Doherty amplifier. Apart from the PAPR effects on the PA efficiency (in the sense of the ratio of useful power to total power), reduced PAPR values are also beneficial to fully exploit the dynamic range of other subsystems, such as the digital-toanalog (D/A) converters, in order to minimize undesired amplitude clippings.

The PAPR in LTE systems has been assessed in both aggregated and non-aggregated channel alternatives [30], and the use of peak reduction (PR) techniques have been studied to reduce the nonlinear effects in the PAs. In a nonaggregated LTE system, and from MATLAB simulations matched to actual FPGA processing results for $20 \mathrm{MHz} 15-$ channel Rev C EVDO, the PAPR for a given CCDF (Complementary Cumulative Density Function) may be reduced by $2 \mathrm{~dB}$ with the use of the peak reduction (PR) techniques. With aggregated-band LTE the PAPR reduction results similar, although higher sporadic peak values are obtained compared to non-aggregated LTE.

As indicated above in multichannel operation the choice between channel bonding and aggregation methods depend on different aspects related with the system performance at the MAC (medium access control) layer. As indicated in Section 2, this decision does not always have a clear answer, depending on the number of users and the kind of traffic. Besides, an additional element to help in the decision-making may be based on the resulting PAPR, according to the kind of multichannel operation: bonding or aggregation. Little work has been made to study this aspect, and nowadays there are no studies indicating if one multichannel strategy is preferable to another from the PAPR perspective, or even if this fact is invariant for taking a design decision. This is the objective of the rest of the paper.

\section{$4 \quad$ Simulation analysis}

\subsection{Scenario}

In the next sections the PAPR is assessed for different types of channel fragmentations. In order to have trustworthy and realistic results, we have selected a practical scenario, based on a standardized radiocommunications system. In particular, the chosen radio standard to carry out the studies has been the ETSI EN 302 217-2-2 V1.4 [17], where the characteristics and requirements for both equipment and antennas are labelled. Simulations details not described in the document [17] have been taken from the CEPT/ERC/ RECOMMENDATION 12-02E [31]. For each channel, the nominal bit rate has been taken according to the one defined for STM-1 (Synchronous Transport Module level1: $155.52 \mathrm{Mbps})$, which together with a Reed-Solomon $\mathrm{RS}(204,188,8)$ - coding produces a rate of 168.7557 Mbps. The modulation format is 128 QAM. The digital upsampling is made by means of two cascaded sub-systems, a root-raised cosine interpolation filter with a roll-off factor of $\alpha=0.16$ and a comb filter. The interpolation factor is 50 (samples per symbol), and the sampling frequency is 1200 $\mathrm{MHz}$. By using a fixed carrier spacing of $28 \mathrm{MHz}$, the basic channel arrangement is compliant with the CEPT recommendation in the band $12.75-13.25 \mathrm{GHz}$, Fig. 1 .

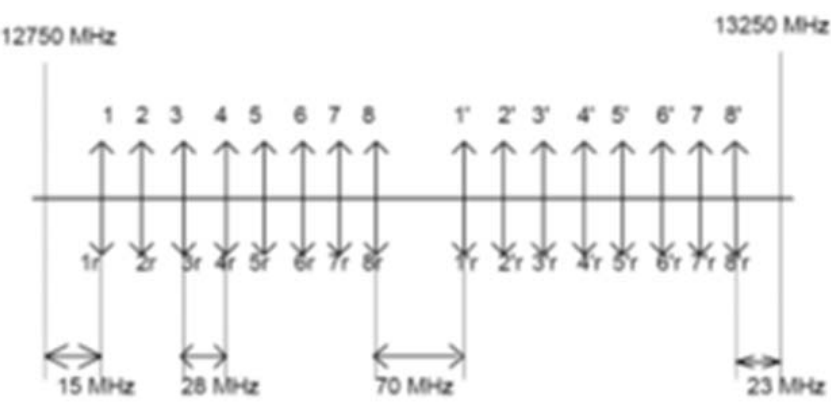

Fig.1 CEPT Radio-frequency channel arrangement in the band $12.75 \mathrm{GHz}$ to $13.25 \mathrm{GHz}$ using the $28 \mathrm{MHz}$ channel spacing (reproduced from [31])

The bandwidth of commercial PAs in this band may range from hundreds of $\mathrm{MHz}$ to some $\mathrm{GHz}$, depending on the PA technology, the stability margin (or, indirectly, the gain) and the power efficiency. In this study, we have assumed two different cases for the PA. First, we have considered that a single PA is used for each set of eight consecutive channels, being necessary two PA to cover the entire band (1-8 and 1'-8' in Fig. 1). And secondly, we have considered a single PA to cover the 16 channels band. The assessed situations have been contiguously-spaced, regularly-spaced and randomly-spaced active channels.

As usual, the PAPR is here defined as 


$$
\operatorname{PAPR}(s(t))=\frac{\max _{0 \leq t \leq T}|s(t)|^{2}}{E\left\{|s(t)|^{2}\right\}}
$$

where $E\{\cdot\}$ denotes the expectation operator. In the discrete time version, the equation becomes:

$$
\operatorname{PAPR}(s(n))=\frac{\max _{0 \leq n \leq L N-1}|s(n)|^{2}}{E\left\{|s(n)|^{2}\right\}}
$$

being $L$ the oversampling factor and $N$ the sequence length.

Raw PAPR measurements place too much emphasis on instantaneous peak values of the signal. So the direct use of the above equations to compute the PAPR can produce wrong results if an instantaneous value of the maximum is due to an anomalous (infrequent) peak, which additionally will make difficult the repeatability of the measurements. To mitigate this fact, in some commercial equipment the peak value is sometimes considered to be the level where 99.8 percent of the waveform is below the maximum peak value, and 0.2 per cent of the waveform is above it. In other measurement devices it is customary to define the signal peak as the magnitude value where the CCDF curve passes through 0.0001 probability $[32,33]$. Also, with the purpose of mitigating the effect of anomalous peaks, we have split the data sequence in several frames in this study, computing the PAPR of each one and lastly computing the mean value of all the PAPRs in order to have a smoothed result with little dependence of infrequent peaks.

On the other hand, the theoretical PAPR of different modulated signals may be very different if it is measured directly over the modulated signal or it is measured after the ISI (inter-symbol interference) filters, whose roll-off factor increases the PAPR as much as the filter slope is approaching ideality. To directly mix PAPR figures taken before and after the ISI filter could produce misinterpretations.

It will be here assumed the PA to be transmitting continuously, so the additional intermodulation and harmonic distortion produced when the PA requires intermittent transmission [34] and occasional switching between channels, won't be considered. Besides, the channels, once selected, are assumed to operate without spectrum handoff.

\subsection{Simulation results}

Physical details of the PA are not included in this study since the objective is just to know the PAPR before it, in order to dimension the back-off requirements when designers have to face its biasing. However, a basic Saleh model of the PA will be later included in the study of the PR techniques, in order to know the resulting EVM deterioration after clipping the PA input signal.

The first simulation results correspond to the case of a PA covering the half band (first 8 channels). In Fig. 2 the case of 8 simultaneously transmitted channels is shown. The resulting PAPR is $13.05 \mathrm{~dB}$.

If only alternated channels are activated (i.e, channels 2,4,6,8), Fig. 3, the smoothed PAPR becomes $12.72 \mathrm{~dB}$. It is noticeable the low PAPR reduction even though the number of active channels has been reduced to the half.
In a similar way, the obtained results for different channel arrangements are summarized in Tables 1 and 2 .
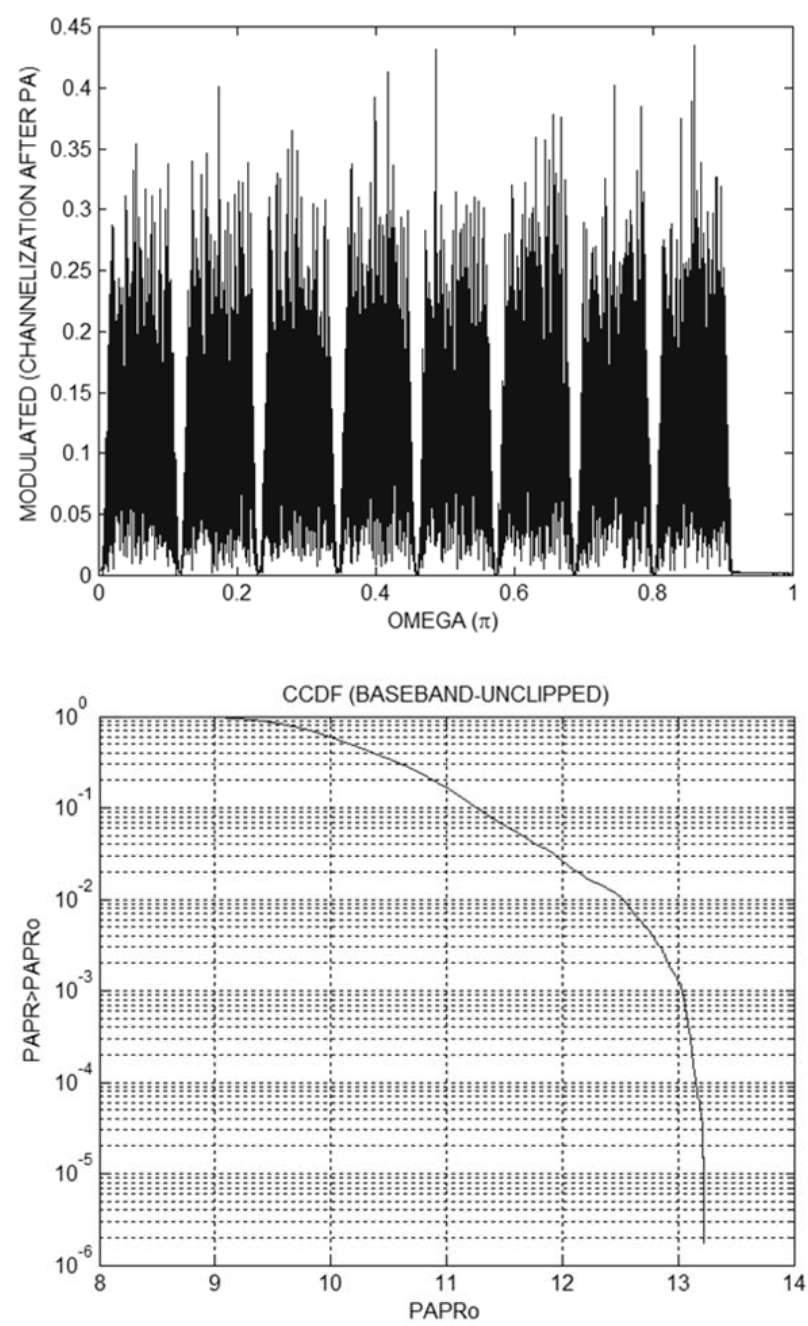

Fig.2 Spectrum (top) linearly scaled -not in dB- and indicated in discrete-time frequency and CCDF curve (bottom) of a simultaneous contiguous active channel transmission.

Setting the CCDF of $10^{-4}$, the results tend to the values indicated in Table 3 , being the PAPR increment within the range of $0.9-1.6 \mathrm{~dB}$ each time the number of channels is doubled. This small increment only depends on the number of channels, not on their relative allocation. So, it is indifferent if they are contiguously, regularly or randomly spaced.

When a broad channel is made by bonding two sets of 8 channels (Fig. 4), the PAPR figures result similar to the ones obtained for one single channel of only $28 \mathrm{MHz}$. This result shows the practical independence on the number of bonded channels used to form the broad one. Hence, from the PAPR viewpoint, the channel bonding option is preferable to channel aggregation: while the first keeps the PAPR practically equal to the value obtained for a single 
channel, the aggregation method increases PAPR as much as the number of aggregated channels also increases.
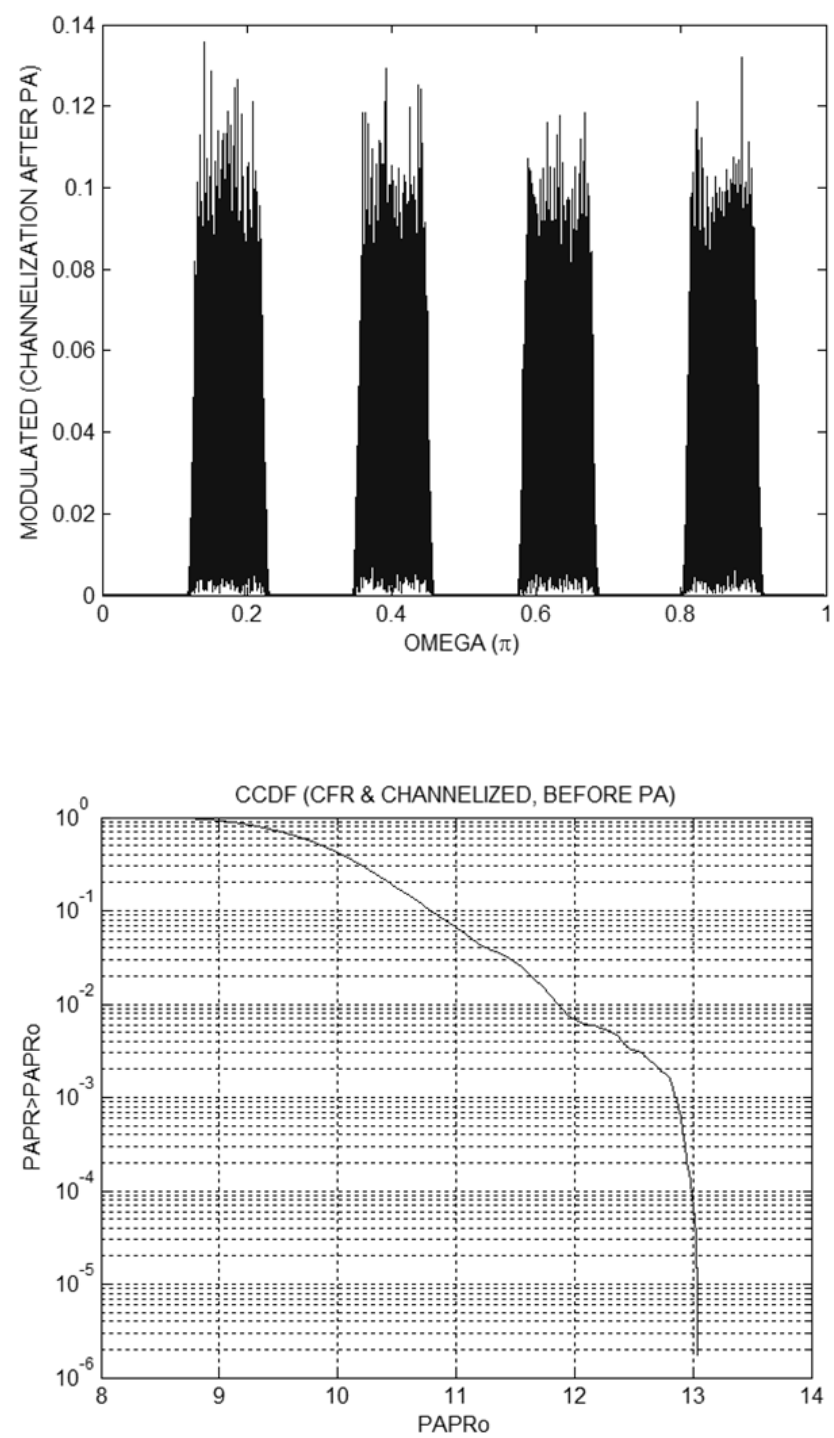

Fig. 3 Spectrum (top) and CCDF (bottom) when only alternated channels are activated
Table 1 Summary of PAPR values for a power amplifier (PA) covering the half-band ( 8 channels bandwidth) and transmitting different active channel combinations

\begin{tabular}{|l|c|c|}
\hline CHANNELS & $\begin{array}{c}\text { NUMERATION } \\
\text { OF ACTIVE } \\
\text { CHANNELS }\end{array}$ & $\begin{array}{c}\text { PAPR } \\
\text { HALF BAND PA }\end{array}$ \\
\hline - Just one & 2 & $10.34 \mathrm{~dB}$ \\
\hline - 2 separated channels. & 2,8 & $11.46 \mathrm{~dB}$ \\
\hline $\begin{array}{l}2 \text { consecutive } \\
\text { channels. }\end{array}$ & $2,3$. & $11.66 \mathrm{~dB}$ \\
\hline $\begin{array}{l}4 \text { consecutive } \\
\text { channels. }\end{array}$ & $1,2,3,4$ & $12.75 \mathrm{~dB}$ \\
\hline $\begin{array}{l}4 \text { alternated channels } \\
\text { - } 3 \text { central channels and } \\
\text { one separated }\end{array}$ & $2,4,6,8$ & $12.72 \mathrm{~dB}$ \\
\hline $\begin{array}{l}\text { 8 simultaneous } \\
\text { channels. }\end{array}$ & $2,4,5,6$. & $12.27 \mathrm{~dB}$ \\
\hline \multicolumn{2}{|l}{} \\
\hline
\end{tabular}

Table 2 Summary of PAPR values for a broadband power amplifier (PA) covering the whole band (16 channels bandwidth) and transmitting different active channel combinations.

\begin{tabular}{|c|c|c|}
\hline CHANNELS & $\begin{array}{c}\text { NUMERATION } \\
\text { OF ACTIVE } \\
\text { CHANNELS }\end{array}$ & $\begin{array}{c}\text { PAPR } \\
\text { PA (covering the } \\
\text { entire band (16 } \\
\text { channels) }\end{array}$ \\
\hline $\begin{array}{c}2 \text { in separated sub- } \\
\text { bands. }\end{array}$ & 2,14 & $11.79 \mathrm{~dB}$ \\
\hline $\begin{array}{c}\text { Two-to-two } \\
\text { alternated. }\end{array}$ & $4,5,12,13$. & $12.37 \mathrm{~dB}$ \\
\hline $\begin{array}{c}4 \text { alternated } \\
\text { channels with } \\
\text { maximal separation. }\end{array}$ & $3,7,11,15$ & $12.53 \mathrm{~dB}$ \\
\hline $\begin{array}{c}8 \text { channels } \\
\text { alternated in sub- } \\
\text { bands }\end{array}$ & $1,3,5,7,9,11,13,15$ & $13.12 \mathrm{~dB}$ \\
\hline $\begin{array}{c}8 \text { simultaneous } \\
\text { channels. }\end{array}$ & $1,2,3,4,5,6,7,8$ & $12.52 \mathrm{~dB}$ \\
\hline $\begin{array}{c}\text { All } 16 \text { channels } \\
\text { active }\end{array}$ & $\begin{array}{c}1,2,3,4,5,6,7,8,9,10 \\
11,12,13,14,15,16\end{array}$ & $15.21 \mathrm{~dB}$ \\
\hline
\end{tabular}


Even in the case of comparing two independent adjacent $28 \mathrm{MHz}$ channels with one bonded channel of $56 \mathrm{MHz}$, the benefit of bonding is about $1.5 \mathrm{~dB}$ in PAPR. And comparing 4 adjacent channels with a bonded one of 112 $\mathrm{MHz}$, the advantage is nearby $2.5 \mathrm{~dB}$. For the entire set of 16 channels it is improved by $5 \mathrm{~dB}$, but this situation is not completely realistic because the selected standard imposes a gap of $70 \mathrm{MHz}$ between each set of 8 channels. To aggregate using two subsets of 8 channels produces PAPR values ranging between $3 \mathrm{~dB}$ and $3.5 \mathrm{~dB}$ higher than bonding them.

Table 3 PAPR for different number of active channels $\quad\left(\mathrm{CCDF}=10^{-4}\right)$

\begin{tabular}{|c|c|}
\hline PAPR & $\begin{array}{c}\text { Number of channels } \\
\text { (one channel: 28 } \\
\text { MHz) }\end{array}$ \\
\hline $10.4 \mathrm{~dB}$ & 1 \\
\hline $11.6 \mathrm{~dB}$ & 2 \\
\hline $12.4 \mathrm{~dB}$ & 4 \\
\hline 13.3 & 8 \\
\hline 14.9 & 16 \\
\hline
\end{tabular}

\section{Peak factor reduction}

Two different peak reduction techniques have been tested: compression (A-law based) and clipping and filtering techniques. The A-law compressor (also used in European standards for audio compression in telephony) follows the relationship:

$y=\left\{\begin{array}{c}y_{\max } \frac{A\left(|x| / x_{\max }\right)}{1+\log _{e} A} \operatorname{sig}(x), \quad 0<\frac{|x|}{x_{\max }} \leq \frac{1}{A} \\ y_{\max } \frac{1+\log _{e} A\left(|x| / x_{\max }\right)}{1+\log _{e} A} \operatorname{sig}(x), \quad \frac{1}{A}<\frac{|x|}{x_{\max }}<1\end{array}\right.$

We have discarded the use of the $\mu$-law because the results obtained with this law have been inferior. Although better, the achieved results by using the A-law have not been outstanding: PAPR may be reduced in the range of 1 to $4 \mathrm{~dB}$ (Fig.6, shows a reduction is $1.5 \mathrm{~dB}$ for the case of a subset of 4 channels), but at the price of vastly increased EVM values (Fig. 5 shows the form of the constellation distortion for a the case of a channel transmitted within a set of 8). For instance, a reduction of $2 \mathrm{~dB}$ in PAPR produces an enlargement of the EVM ranging between 7 and $30 \%$ depending on the number of active channels.
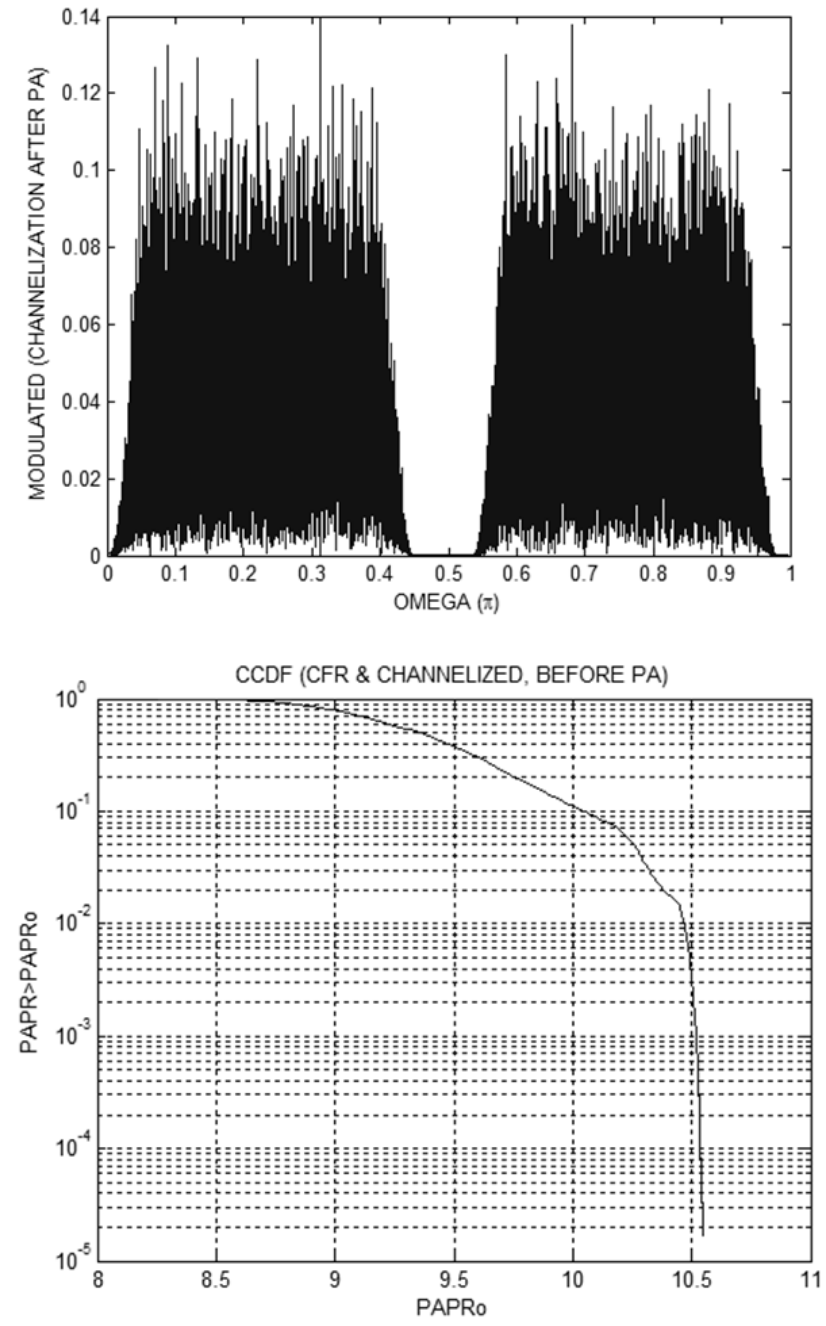

Fig. 4 Bonded groups of $8+8$ channels. (top): spectrum, (bottom): CCDF (PAPR)

Channel aggregation is the strategy that produces the worst EVM values for a given PAPR reduction. Conversely, channel bonding produces lower EVM degradation, even though the resulting values are not acceptable because of the specification strictness in modern communication standards.

In the clipping and filtering technique [35] the clipped signal $\hat{x}(t)$ is expressed by the following relationship:

$$
\widehat{x}(t)=\left\{\begin{array}{cc}
\lambda \cdot e^{j \varphi(t)}, & |x(t)|>\lambda \\
x(t), & |x(t)| \leq \lambda
\end{array}\right.
$$

where $x(t)$ is the original signal, $\lambda$ is the clipping level and $\varphi(t)$ is the phase of the original signal. The clipping noise is:

$$
x_{c}(t)=x(t)-\hat{x}(t)
$$

which, once filtered, becomes:

$$
f(t)=\text { filtered }\left(x_{c}(t)\right)
$$


resulting the clipped and filtered signal:

$$
y(t)=x(t)-\beta \cdot f(t)
$$

being $\beta$ a weighting factor (empirical) of the clipping noise. Results are too sensitive to the chosen values of $\lambda$ and $\beta$. Low values of $\lambda$ and high values of $\beta$ produce a larger peak clipping (PAPR shrinking), at the price of increasing the EVM. The use of this technique (originally designed for OFDM systems) in the multichannel scenario here studied produces inacceptable increments of the EVM. Even for small PAPR reductions (of $1.5 \mathrm{~dB}$ or lower), the EVM figure increases from $3 \%$ to $20 \%$, depending on $\lambda$ and $\beta$.

The EVM (per channel) is being reduced as the number of aggregated channels increases. Hence, the clipping and filtering strategy produces an inverse conclusion with regards of the A-law compression: concerning the EVM values, the channel aggregation is the better choice to use the clipping and filtering technique.

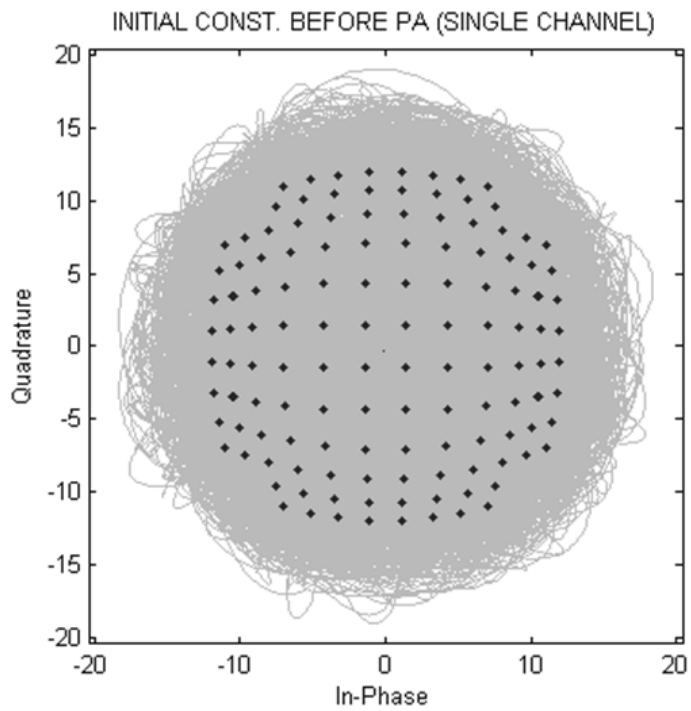

Fig. 5 Effects of the A-law compression on the constellation of a single channel (transmitted in a set of 8).

In Fig. 7 we can see how the clipping noise produces a blurred constellation. Fig. 8 shows the simulated results for $\lambda=0.5$ and $\beta=0.7$, using a Hamming filter to remove the clipping noise. In Fig. 9 the CCDF and the spectrum when each set of 8 channels has been bonded in one broadband channel. Regarding PAPR reduction and the clipping noise disturbance in the adjacent channels, both aggregation and bonding techniques look similar (Figs 8 and 9). The main difference is the above-mentioned advantage of aggregation regarding the EVM degradation.

\section{Conclusion}

A comparative study of the channel bonding and channel aggregation by using a standardized radio link as the

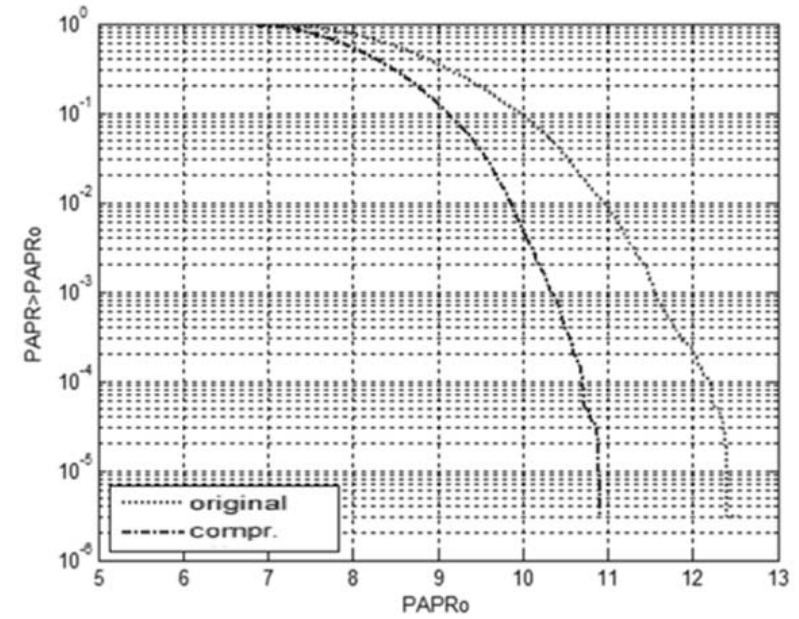

Fig. 6 Effect of the A-law compression on the CCDF curve of a transmitted subset of 4 channels

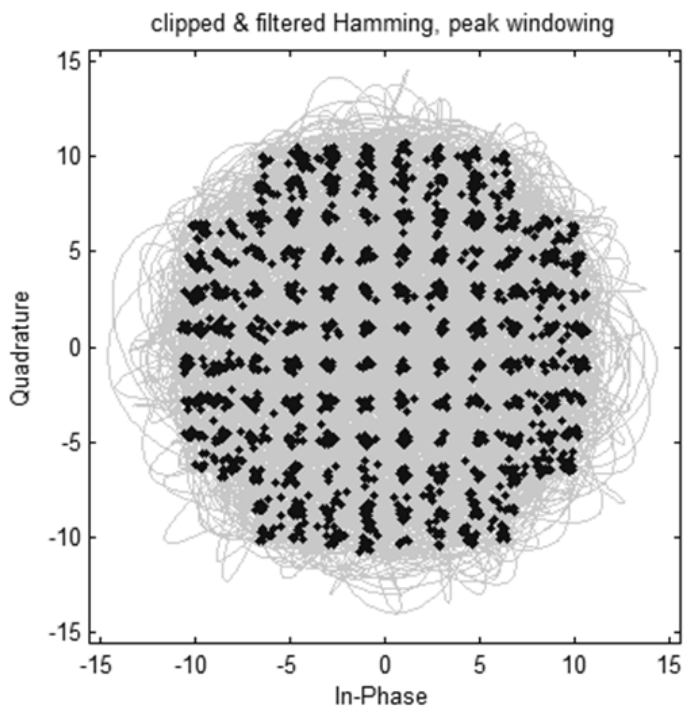

Fig. 7 Blurred constellation (clipping noise).

conducting model to assess both multichannel strategies has been made. As the main result of the comparison, the PAPR favors the use of the channel bonding, strategy with advantages of up to $3.5 \mathrm{~dB}$.

In channel aggregation, we have obtained a conclusion in coherence with the results published in [36] for NC-OFDM (non-contiguous OFDM) transmission. In contiguous and regularly-spaced active channels (which are the subcarriers in [36]), the PAPR distribution is practically the same, independently of the channels arrangement. Hence, the PAPR increment depends only on the number of channels, not on the relative channel allocation, and it is augmented from 0.9 to $1.6 \mathrm{~dB}$ each time that the number of channels is doubled. However, and dissimilarly to [36], in the case of randomly-spaced active channels we keep the same conclusion, not differing the PAPR values from the ones obtained in contiguous or regularly spaced channel 
organizations. As a conclusion, the PA back-off has to be only increased by $3 \mathrm{~dB}$ when the number of active channels changes from 1 to 8 , being the power efficiency clearly proportional to the number of channels and having little effect their relative allocation along the band.
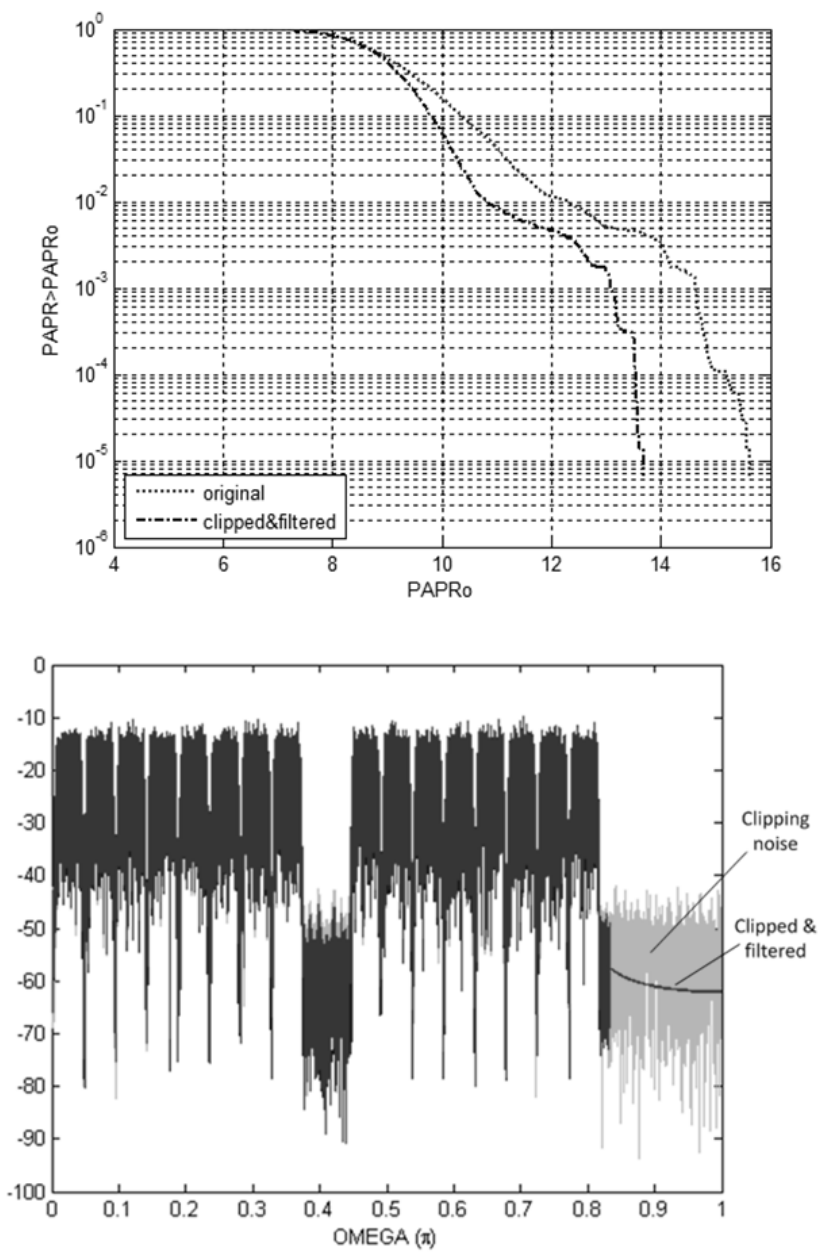

Fig. $8 \mathrm{CCDF}$ reduction and simulated spectrum (ACPR) for $\lambda=0.5$ and $\beta=0.7,16$ Channels. (top): CCDF (PAPR), (bottom): spectrum (ACPR).

Additionally, two CFR techniques have been tested, one based on a compression (A-law) and other in the clipping and filtering. With the compression the PAPR may be reduced from 1 to $4 \mathrm{~dB}$, while the clipping and filtering technique reduces 1 or $2 \mathrm{~dB}$. The first produces an enlargement of the EVM from 7 and $30 \%$ for a reduction of $2 \mathrm{~dB}$ in PAPR, being the EVM results better in the case of channel aggregation. The clipping and filtering technique produces increases in the EVM figure from 3\% to $20 \%$, being the channel bonding the option that yields lower PAPR in the filtered signal. Anyway, the EVM degradation makes inadvisable the application of CFR strategies for most of the modern communication standards non OFDM based. When using the clipping and filtering technique, a partial solution to reduce the PAPR and alleviating the undesired EVM increase is to enlarge the
BW of the noise filters, applicable when more clipping noise in the adjacent channels might be allowed.

The conclusions here obtained have been carried out with the objective to bring new elements at the PHY level to optimize the algorithms for multichannel assignment, thus complementing other considerations already published at the MAC level and, consequently, allowing the simultaneous attention of aspects from both MAC and PHY layers.
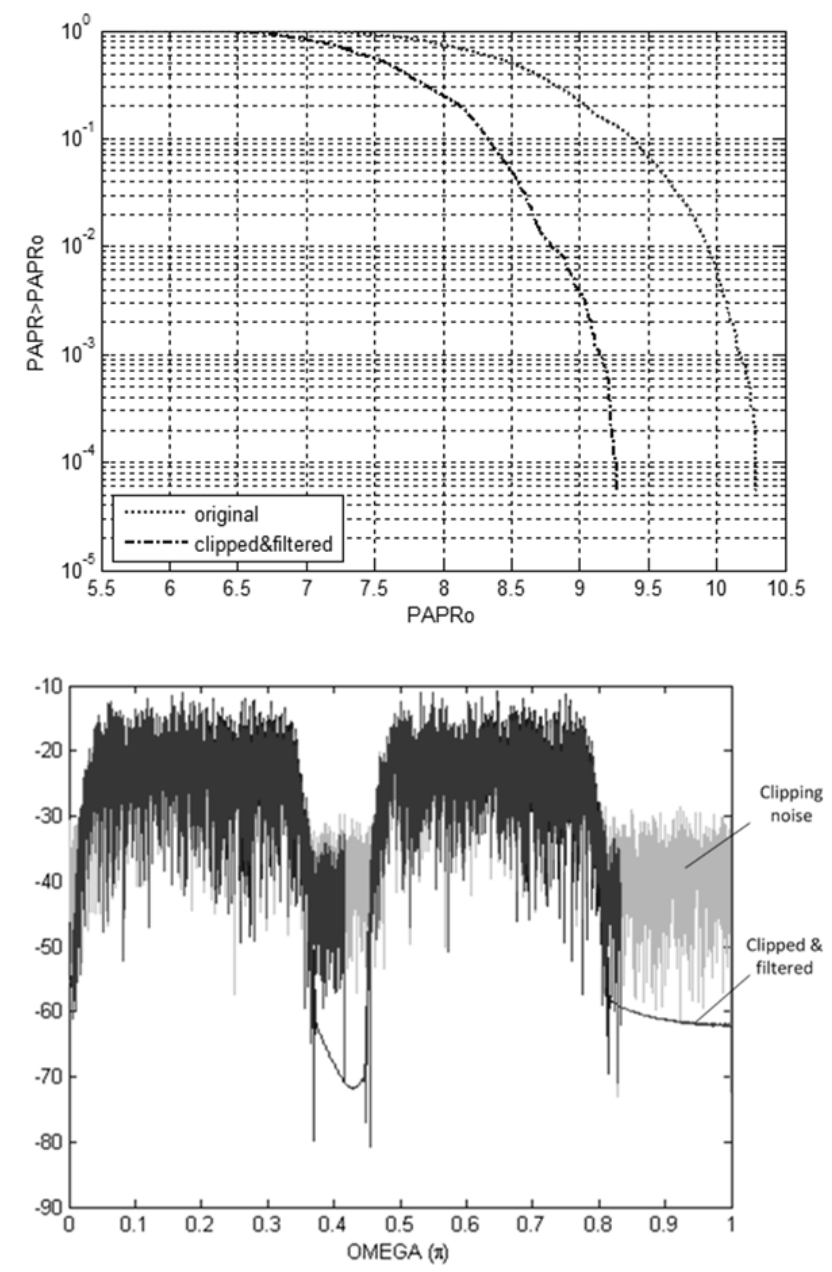

Fig. $9 \mathrm{CCDF}$ (top) and spectrum (bottom) of the result of bonding $8+8$ channels, $\lambda=0.4$ and $\beta=0.7$.

\section{Acknowledgment}

This work was partially supported by the Spanish Ministry of Economy and Competitiveness (MINECO) under project TEC2014-58341-C4-3-R, and by the Secretary for Universities and Research of the Government of Catalonia, under grant 014 SGR 1103.

\section{References}

1 Manzoor Ahmed Khan, et al: Game Dynamics and Cost of Learning in Heterogeneous 4G Networks. IEEE Journal on Selected Areas in Communications vol. 30, n.1: pp198-213 2012. 
2 Wannstrom, J. LTE-Advanced. [Online]. Available: http://www.3gpp.org/lte-advanced, accessed 15 October 2014.

3 Damnjanovic, A., Montojo, J., Wei, Y., et al. A survey on $3 \mathrm{GPP}$ Heterogeneous Networks. IEEE Wireless Communications, vol. 18, n. 3, pp 10-21, 2011.

4 David López-Pérez, et al: On Distributed and Coordinated Resource Allocation for Interference Mitigation in SelfOrganizing LTE Networks. IEEE Transactions on. Networking, vol. 21, n.4. pp 1145-1158. 2013.

5 Pedro B. F. Duarte, Zubair Md. Fadlullah, Athanasios V. Vasilakos, Nei Kato: On the Partially Overlapped Channel Assignment on Wireless Mesh Network Backbone: A Game Theoretic Approach. IEEE Journal on Selected Areas in Communications vol. 30, n.1. pp 119-127. 2012.

6 Berlemann, L., Mangold, S. Cognitive Radio and Dynamic Spectrum Access. John Wiley \& Sons, 2009.

$7 \mathrm{Xu}, \quad$ L., Yamamoto, K., Yosrhida, S. Performance Comparison between Channel-Bonding and Multi-Channel CSMA. Proc. IEEE Communications and Networking Conference, WCNC, Hong Kong, 2007.

8 Sang-Seon Byun, et al: Computation of an Equilibrium in Spectrum Markets for Cognitive Radio Networks. IEEE Tranactions on Computers, vol. 63, n. 2. pp 304-316. 2014.

9 Goratti, L., Baldini, G., Rabbachin. An urn occupancy approach for cognitive radio networks in DTVB white spaces. Telecommunication Systems (2014) 56, pp:229-244. DOI 10.1007/s11235-013-9832-9

10 Alireza Attar, et al: A Survey of Security Challenges in Cognitive Radio Networks: Solutions and Future Research Directions. Proceedings of the IEE, vol. 100, n. 12. pp 31723186. 2012.

11 Z.Sheng et al: A survey on the ietf protocol suite for the internet of things: standards, challenges, and opportunities. IEEE Wireless Communications, vol. 20, n.6, pp 91-98. 2013.

12 M. Chen, et al., A Survey of Recent Developments in Home M2M Networks. IEEE Communications Surveys \& Tutorials, vol. 16, no. 1, pp. 98-114, First Quarter 2014.

13 Xiaofei Wang, et al: A Survey of Green Mobile Networks: Opportunities and Challenges. Mobile Networks and Applications, vol. 17, n.1. pp 4-20. 2012.

14 Kai Han, Jun Luo, Yang Liu, A.V. Vasilakos: Algorithm design for data communications in duty-cycled wireless sensor networks: A survey. IEEE Communications Magazine, vol. 51, n. 7. 2013.

15 A. V. Vasilakos, et al: Delay tolerant networks: Protocols and applications. CRC Press, 2012

16 Y. Zeng, et al: Directional routing and scheduling for green vehicular delay tolerant networks". Wireless networks. Springer. Vol. 19, n. 2, pp 161-173. 2013.

17 Fixed Radio Systems; Characteristics and requirements for point-to-point equipment and antennas; Part 2-2: Digital systems operating in frequency band. Harmonized European Standard ETSI. EN 302 217-2-2 V1.4.1 (2010-07). 2010.

18 M. Youssef, et al: Routing Metrics of Cognitive Radio Networks: A Survey, IEEE Communications Surveys and Tutorials, vol. 16, n.1. pp 92-109. 2014.

19 Martorell, G., Riera-Palou, F., Femenias, G. Modeling fast link adaptation-based $802.11 \mathrm{n}$ distributed coordination function. Telecommunication Systems (2014) 56. pp:215227. DOI 10.1007/s11235-013-9831-x

20 Joshi, S., Pawełczak, P,. Čabrić, D., Vilasenor, J. When Channel Bonding is Beneficial for Opportunistic Spectrum Access Networks. IEEE Transactions on Wireless Communications, vol. 11, n.11, pp. 3942 - 3956, 2012.
21 Jiao, L., Pla, V., Li, F.Y. Analysis on Channel Bonding/Aggregation for Multi-channel Cognitive Radio Networks. Proc. European Wireless 2010, EWC, pp. 468474, Lucca, Italy, 2010.

22 Balapuwaduge I.A.M. Performance Evaluation of Channel Aggregation Strategies in Cognitive Radio Networks with Queues. [Online]. Univ. of Agder, Norway, pp. 81-90, Available:

http://brage.bibsys.no/xmlui/handle/11250/92952/discover. Accessed 15 October 2014.

23 Tigang Jiang, et al: QoE-Driven Channel Allocation Schemes for Multimedia Transmission of Priority-Based Secondary Users over Cognitive Radio Networks. IEEE Journal on Selected Areas in Communications, vol. 30, n. 7. pp 1215-1224. 2012.

24 Vasu Chakravarthy, et al: Novel overlay/underlay cognitive radio waveforms using SD-SMSE framework to enhance spectrum efficiency- part i: theoretical framework and analysis in AWGN channel. IEEE Transactions on Communications vol. 57, n. 12. pp 3794-3804. 2009.

25 Salameh, H.B., Krunz, M., Manzi, D. Spectrum Bonding and Aggregation With Guard-band Awareness in Cognitive Radio Networks. IEEE transactions on Mobile Computing . vol. PP, n 99, 2013.

26 Jiao, 1., Li, F.Y., Pla, V. Modeling and Performance Analysis of Channel Assembling in Multichannel Cognitive Radio Networks with Spectrum Adaptation, IEEE Transactions on Vehicular Technology, vol.61, n.6, July 2012.

27 Anand, S., Hong, K., Sengupta, S., Chandramouli, R. Is Channel Fragmentation/Bonding in IEEE 802.22 Networks Secure? IEEE International Conference on Communications. ICC2011, pp. 1-5, Kyoto, June 2011.

28 Cordeiro, C., Ghosh, M. Channel Bonding versus Channel Aggregation, IEEE 802.22 WG document no. 22-06-010800-0000, July 2006.

29 Alsabbagh, E,. Yu, H., Gallagher, K. 802.11ac Design Considerations for Mobile Devices. Microwave Journal, vol 56, n.2, pp 80- 88, February 2013.

30 Mc Callister. Ideal Amplification of Broadband Signals. International Journal on Microwave and Wireless Technologies. vol. 5, pp 179 - 186, April 2013.

31 Harmonized Radio Frequency Channel Arrangements for Analogue and Digital Terrestrial Fixed Systems Operating in the Band $12.75 \mathrm{GHz}$ to $13.25 \mathrm{GHz}$, CEPT/ERC/RECOMMENDATION 12-02E, Working Group "Spectrum Engineering", June 2007.

32 McCune, E (2010), Modern Wireless Signals. [Online], Available: http://www.bessernet.com/BesserBlog/?p=179, accessed 15 October 2014.

33 Characterising Digitally Modulated Signals with CCDF Curves. Agilent Technologies, Application note. 2000.

34 Watkins, G., Wang, S. The Impact of Power Amplifier TurnOn Characteristics in Cognitive Radio Networks. Microwave Journal, Vol.57, No.3, pp 86- 92, March 2014.

35 Wang, L., Tellambura, C. A Simplified Clipping and Filtering Technique for PAR Reduction in OFDM Systems. IEEE Signal Processing Letters, Vol. 12, N.. 6, pp. 453-456, June 2005.

36 Rajbanshi, R., Wyglinski, A.M, Minden, G.J. Peak-toAverage Power Ratio Analysis for NC-OFDM Transmissions. Proc. IEEE 66th Vehicular Technology Conference, VTC-2007, Baltimore, Maryland USA. 